\title{
Relativistic motion of an Airy wave packet in a lattice potential
}

\author{
C. E. Creffield \\ Departamento de Física de Materiales, Universidad Complutense de Madrid, E-28040 Madrid, Spain
}

(Received 21 August 2018; published 7 December 2018)

\begin{abstract}
We study the dynamics of an Airy wave packet moving in a one-dimensional lattice potential. In contrast to the usual case of propagation in a continuum, for which such a wave packet experiences a uniform acceleration, the lattice bounds its velocity, and so the acceleration cannot continue indefinitely. Instead, we show that the wave packet's motion is described by relativistic equations of motion, which surprisingly arise naturally from evolution under the standard nonrelativistic Schrödinger equation. The presence of the lattice potential allows the wave packet's motion to be controlled by means of Floquet engineering. In particular, in the deep relativistic limit when the wave packet's motion is photonlike, this form of control allows it to mimic both standard and negative refraction. Airy wave packets held in lattice potentials can thus be used as powerful and flexible simulators of relativistic quantum systems.
\end{abstract}

DOI: 10.1103/PhysRevA.98.063609

\section{INTRODUCTION}

In 1979 Balasz and Berry [1] demonstrated that the Airy function is a nondiffracting solution of the Schrödinger equation of a free particle in one dimension. As well as being nonspreading, this solution also has the unique property of appearing to accelerate at a constant rate, even in the absence of an external force. Following on from this result, a great deal of work has been done both theoretically and experimentally to study this kind of behavior, with a particular eye to harnessing its unusual properties for applications such as particle manipulation [2,3], optical routing [4], and microscopy [5]. The first experimental observations of Airy wave packets were made in an optical system [6], making use of the correspondence between the paraxial diffraction equation and the Schrödinger equation. Later work showed how Airy wave packets of free electrons could be generated [7] and, more recently, it has been suggested to use systems of Bose-Einstein condensates $[8,9]$, in order to generate self-accelerating matter waves.

The majority of studies have concentrated on the continuum case. Lattice systems, however, represent a fascinating arena to investigate and make use of the dynamics of Airy wave packets. Such systems must clearly reproduce the continuum behavior as the lattice spacing is reduced to zero, but can be expected to show novel features arising from the interplay between the spatial discretization and the selfacceleration effect. In particular, systems of ultracold atoms held in optical lattice potentials [10] are excellent candidates for studying these effects, due to their high degree of quantum coherence and their controllability [11]. Such systems have already been used as idealized lattice simulators to emulate the quantum dynamics of condensed-matter systems, such as the Hofstadter butterfly [12,13], the direct observation of Bloch oscillations [14], and Veselago optics [15].

In this work, we investigate the dynamics of an Airy wave packet moving on a tight-binding lattice. We will first see that the initial behavior of this system duplicates that of the continuum case, with the wave packet undergoing a constant acceleration. For longer times, however, this acceleration reduces towards zero and the velocity of the wave packet saturates to a maximum value. This is a direct effect of the spatial discretization, since the speed of propagation in the lattice is bounded by the lattice dispersion relation. As a consequence the kinematics of the system is described very accurately by the formalism of special relativity, with the lattice group velocity playing the role of the "speed of light." Rather unexpectedly, this relativistic description emerges spontaneously from the dynamics of the standard nonrelativistic Schrödinger equation. This contrasts, for example, with the behavior of Gaussian wave packets in a tilted lattice; these are also subject to a constant accelerating force, but instead undergo Bloch oscillation $[16,17]$. We then show how Floquet engineering [18] allows us to manipulate the system's dispersion relation, and thus control the propagation of the wave packet. This demonstrates how using Airy wave packets in lattice potentials is a convenient and powerful method to simulate relativistic quantum systems.

\section{AIRY WAVE PACKET}

\section{A. Self-acceleration}

We begin by considering the Schrödinger equation for a particle of mass $m$, moving in a one-dimensional system in the absence of any external potentials

$$
i \hbar \frac{\partial \psi}{\partial t}=-\frac{\hbar^{2}}{2 m} \frac{\partial^{2} \psi}{\partial x^{2}}
$$

where $x$ represents a dimensionless spatial coordinate and $t$ is the corresponding time coordinate. For convenience we shall now set $m$ and $\hbar$ equal to one. As was shown in Ref. [1], a solution of Eq. (1) is given by the Airy function [19]

$$
\psi(x, t)=\operatorname{Ai}\left[x-(t / 2)^{2}\right] \exp \left[i(x t / 2)-i t^{3} / 12\right],
$$

which can be readily verified by direct substitution. We plot the corresponding probability density for $t=0$ in 


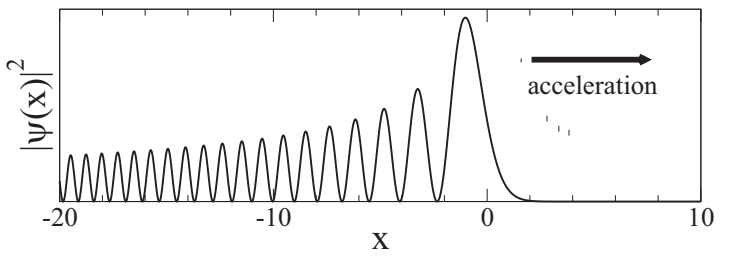

FIG. 1. Probability density of the Airy wave packet given in Eq. (2) at $t=0$. For positive values of $x$ the probability density drops sharply with distance, while for negative $x$ the function has a decaying oscillatory behavior. The slowness of this decay (3) means that the Airy function is not normalizable. Under the action of the Hamiltonian in Eq. (1) this wave packet will accelerate to the right at a constant rate.

Fig. 1. For $x>0$, the Airy function decays rapidly with the form $\operatorname{Ai}(x) \sim \exp \left[-2 x^{3 / 2} / 3\right] / x^{1 / 4}$, which produces the welldefined wave front at the right of the wave packet. Conversely, for large negative values of $x$, the Airy function has a decaying oscillatory form [20]

$$
\operatorname{Ai}(x \rightarrow-\infty) \sim \frac{1}{\sqrt{\pi}|x|^{1 / 4}} \sin \left(2|x|^{3 / 2} / 3+\pi / 4\right) .
$$

From Eq. (2) it is clear that the probability density $|\psi(x, t)|^{2}$ preserves its shape over time, and that it follows a parabolic trajectory $x(t)=t^{2} / 4$, that is, it appears to undergo a constant acceleration of $a=1 / 2$ in the system of units we use. Although this nonintuitive result would appear to violate the Ehrenfest theorem, this is not in fact the case. As can be seen from Eq. (3), the Airy wave function is not $L^{2}$ integrable, and so its center of mass is undefined. Consequently we cannot interpret the acceleration as the response of the system's center of mass to a force. If instead, however, we focus our attention on some specific points of the wave function, such as, for example, the maxima of the probability distribution, we will indeed see the locations of these points moving along parabolic trajectories in the $x-t$ plane (see Fig. 2 for small values of $t$ ). For the remainder of the paper we will concentrate on the motion of the first, and highest intensity, peak of the wave function, which at $t=0$ is centered on $x \simeq-1.019$, and use its position to calculate the wave packet's velocity and acceleration. As this acceleration arises spontaneously, in the absence of any external potentials, we shall refer to it as "self-acceleration."

\section{B. Normalization and diffraction}

As the probability density is not normalizable, a true Airy wave packet can clearly not be prepared in experiment. One option is to use an aperture-limited version of the function, by simply truncating the spatial coordinate to run over a large, but finite, range of values. Smoother aperture functions can also be used to render the wave function normalizable, for example, an exponential function, $\psi(x, t=0)=\operatorname{Ai}(x) \exp (\gamma x)$, where $\gamma$ is a small, positive constant. The Fourier transform of this function is given by

$$
\tilde{\psi}(k) \propto e^{-\gamma k^{2}} e^{i k^{3} / 3},
$$

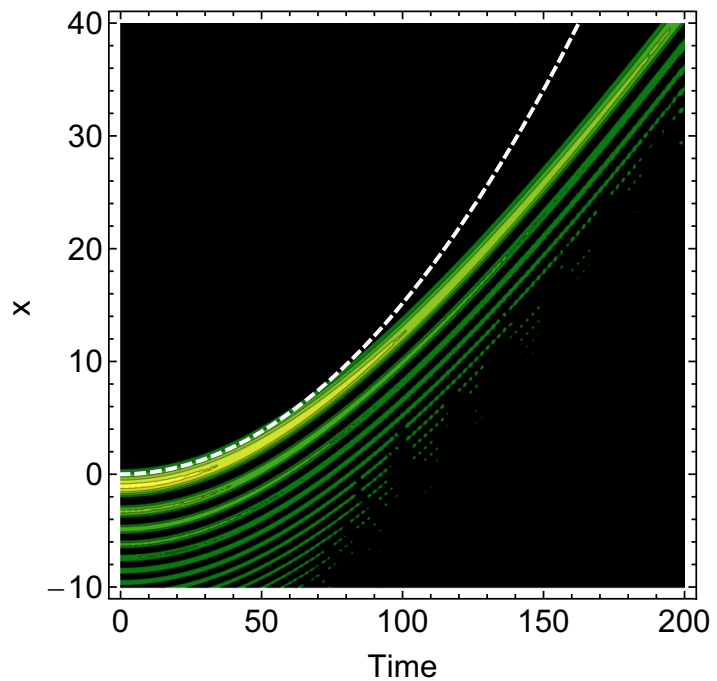

FIG. 2. Trajectory of an Airy wave packet on a lattice. The system is initially prepared in an Airy state, and evolves under the action of the lattice Hamiltonian (5). Initially the peaks in the wave packet move along parabolic trajectories corresponding to a uniform positive acceleration, marked by the white dashed line. At longer times, however, the acceleration of the wave packet decreases as it approaches relativistic speeds. Note that the amplitudes of the peaks decrease with time due to wave-packet spreading; this occurs because the Airy wave packet is aperture limited (see Sec. IIB). Lattice discretization: $\Delta x=0.2$.

where the cubic phase term arises from the Fourier transform of the Airy function itself. This provides a particularly convenient way to synthesize aperture-limited Airy functions in optical systems [6], by simply imprinting a cubic phase on a Gaussian beam using a phase mask, and then making an optical Fourier transform of the result. An analogous technique is also possible for matter-wave optics [7,8] to engineer the appropriate wave function in Fourier space, or alternatively amplitude and phase masks can be used to generate the wave function directly in real space [8].

Truncating the Airy function, and also introducing a spatial discretization, both have the effect that the wave function is no longer diffraction free, and so broadens with time. This sets a finite lifetime for the self-acceleration phenomenon, after which it will break down. This lifetime, over which the Airy wave packet approximately maintains its form, is known as the Airy zone [21]. The majority of the results we report below were obtained by simply limiting the range of the spatial coordinate, and in each case it was verified that the range of $x$ used was sufficiently large that the results were insensitive to it over the time intervals considered. The simulations were also repeated using an exponential aperture function for various values of $\gamma$ as a further check of the stability of the observed effects.

\section{Lattice Airy wave packet}

Instead of allowing the particle to move in free space as in Eq. (1), we now impose a lattice potential $V(x)=V_{0} \cos ^{2} k x$. In ultracold atom experiments, this can be conveniently done by superposing two counterpropagating laser beams [22] to 
create an optical lattice potential whose depth, $V_{0}$, is proportional to the laser intensity. For sufficiently deep optical lattices, the atoms will localize in the potential minima, and can accordingly be described in a basis of site-localized Wannier functions. In this case the dynamics of the atoms can be accounted for well by retaining only the hopping matrix element $J$ that connects a site to its nearest neighbors [11], yielding the lattice Hamiltonian

$$
H_{\text {latt }}=-J \sum_{j=-M}^{N}\left(a_{j+1}^{\dagger} a_{j}+\text { H.c. }\right) \text {. }
$$

Here $a_{j}\left(a_{j}^{\dagger}\right)$ are the usual bosonic annihilation (creation) operators acting on lattice site $j$. The hopping amplitude $J$ is related to the parameters of the continuum Hamiltonian as $J=\hbar^{2} /\left(2 m \Delta x^{2}\right)$, where $\Delta x$ is the lattice spacing which relates the $x$ coordinate to the lattice site, $x=j \Delta x$. Henceforth we will use $J$ as the unit of energy and frequency, and measure time in units of $J^{-1}$.

\section{RESULTS}

\section{A. Motion of the lattice Airy wave packet}

In Fig. 2 we show the movement of a wave packet initialized in the state $\psi(x)=\operatorname{Ai}(x)$, where $x$ is a discretized spatial coordinate with $\Delta x=0.2$, under the time evolution provided by the lattice Hamiltonian (5). At $t=0$ we see the series of peaks in the probability density produced by the $x$ dependence of the Airy function, with the largest peak lying at $x \simeq-1$. For small values of $t(t<80)$, the peaks in the probability density move along trajectories which to a good degree of accuracy are parabolic. We emphasize that this effect is occurring in the absence of any accelerating potentials, and so corresponds to the wave packet undergoing a constant self-acceleration.

For longer times, however, the movement of the peaks clearly begins to deviate from the parabolic behavior, with their location showing a slower, linear dependence on time. This contrasts with the behavior of the continuum Airy wave packet, which would continue to accelerate indefinitely. To see the reason for this discrepancy we can first note that the lattice Hamiltonian (5) can be straightforwardly solved in momentum space

$$
\tilde{H}_{\text {latt }}=-2 J \cos k \sum_{k} a_{k}^{\dagger} a_{k},
$$

giving the dispersion relation $E(k)=-2 J \cos k$. For small $k$ this reproduces the dispersion relation of a free particle $E_{\text {free }}=k^{2} / 2$, but unlike the free particle case, $E(k)$ is limited to a finite range. As a consequence, the group velocity, $v_{g}=$ $2 J \sin k$, has a maximum value of $2 J$, and so the system can only support excitations up to this maximum velocity $v^{\text {max }}$. This is a specific instance of the Lieb-Robinson bound [23]. This motivates us to employ a relativistic description of the system, with this maximum velocity playing the role of the speed of light.

\section{B. Relativistic description}

There is a frequent misconception that the case of a body subject to a constant acceleration cannot be treated within special relativity, but instead necessitates the use of general relativity. This, however, is not the case; special relativity is completely capable of describing such motion [24]. We wish to relate the kinematic quantities measured by an observer at rest with respect to the laboratory (or lattice) with those measured in a uniformly accelerated frame (the instantaneous rest frame of the Airy wave packet). If we denote the (constant) proper acceleration by $\alpha$, then it is a standard textbook exercise [25] to show that the quantities measured in the laboratory frame are given by

$$
\begin{gathered}
a(t)=\alpha\left(1+(\alpha t / c)^{2}\right)^{-3 / 2}, \\
v(t)=\alpha t / \sqrt{1+(\alpha t / c)^{2}}, \\
x(t)=\left(c^{2} / \alpha\right)\left(\sqrt{1+(\alpha t / c)^{2}}-1\right),
\end{gathered}
$$

where $c$ represents the speed of light, and we have imposed the initial conditions $x(0)=v(0)=0$.

In the nonrelativistic limit $\alpha t \ll c$ it can readily be seen that these expressions reduce to the familiar results of Newtonian mechanics, and in particular that $x=1 / 2 \alpha t^{2}$. In general, we can cast Eq. (9) in the more revealing form,

$$
\left(\alpha x / c^{2}+1\right)^{2}-(\alpha t / c)^{2}=1,
$$

to show that the particle follows a hyperbolic trajectory in space-time [26]. The asymptotes of this trajectory are the two straight lines $x= \pm c t$, which form the light cone for this object. The slopes of these lines bound the velocity of the wave-packet velocity (8) such that it never exceeds $c$, but approaches it asymptotically with time.

In Fig. 3 we show the motion of the first peak of the Airy wave packet, for the same parameters as in Fig. 2. The data points in Fig. 3(a) are the motion of the peak obtained from the numerical time integration of the system, while the solid line is the relativistic result for $x(t)$, where $\alpha$ and $c$ were obtained from a two-parameter fit of the numerical data to Eq. (9). The agreement between the data and the fit is excellent. The fit parameters are given in the first line of Table I, and we can see that the obtained value of $c$ is indeed in good agreement with the theoretical value of $v^{\max }=2 \mathrm{~J}$. We also show in this figure the parabolic behavior that would be obtained in the nonrelativistic case, which clearly emphasizes the difference between the relativistic discrete case and the nonrelativistic continuum behavior.

Below in Fig. 3(b) we compare the velocity of the main peak, as calculated by the time derivative of its location, with the relativistic prediction of Eq. (8). Again the agreement is seen to be excellent. The maximum lattice group velocity of $2 J$ is also plotted, and the asymptotic saturation of the peak's velocity to this value is clearly evident. Finally, in Fig. 3(c) we show the acceleration of the wave packet, as measured in the lattice rest frame. As the velocity of the wave packet approaches a significant fraction of $c$, this acceleration reduces smoothly to zero. 


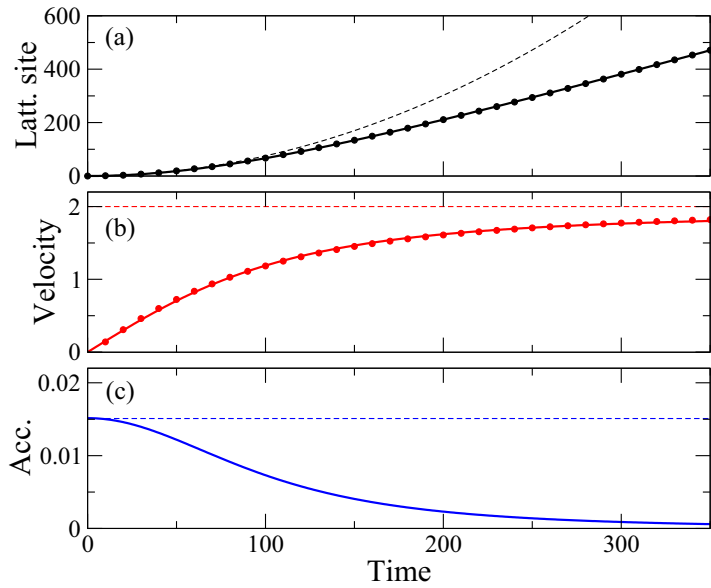

FIG. 3. Relativistic motion of the lattice Airy wave packet, obtained by a numerical simulation of the lattice system. (a) Symbols denote the lattice site occupied by the first peak of the wave packet. Initially this position increases quadratically with time, shown by the dashed line, but for $t>100$ the increase becomes slower, tending towards a linear rise. The solid line shows the relativistic prediction (9), where the values of $c$ and $\alpha$ are obtained from a two-parameter fit to the data points. The fit is excellent. (b) Velocity of the first peak of the lattice Airy function. Symbols show that values obtained from the numerical time derivative of the data from the simulation, while the solid line shows the theoretical result (8). Initially the velocity rises linearly, but flattens off as it begins to approach the maximum lattice velocity $v^{\max }$, shown with the dashed line. (c) Acceleration observed in the rest frame of the lattice. For small times the acceleration is constant, but drops as the wave packet enters the relativistic regime. The dashed line indicates the proper acceleration, $\alpha$, which remains constant.

\section{Fitting and scaling}

In Table I we show values obtained for $\alpha$ and $c$ by fitting the numerical results for different lattice spacings to Eq. (9). Clearly, as $\Delta x$ is reduced, the value of the proper acceleration decreases as well. For $\Delta x=0.05$, the smallest lattice spacing considered, the acceleration was so small that the wave packet did not enter the relativistic regime over the time interval considered. The length of this time interval was limited by the spreading of the wave packet, produced by the aperture

TABLE I. Values of $\alpha$ and $c$ obtained by a two-parameter fit to Eq. (9) for various values of the lattice spacing $\Delta x$. The acceleration $\alpha$ rapidly reduces as the lattice becomes finer, and for the smallest value of $\Delta x$ the wave packet barely entered the relativistic regime in the time span considered. As a result the two-parameter fit was unstable and $\alpha$ was simply evaluated from a fit to a parabola. In all other cases the value obtained for $c$ is in good agreement with the theoretical value of $v^{\max }=2 J$.

\begin{tabular}{lcc}
\hline \hline$\Delta x$ & $c$ & $\alpha$ \\
\hline 0.20 & 1.90 & 0.0153 \\
0.15 & 1.85 & 0.0065 \\
0.10 & 1.81 & 0.0020 \\
0.05 & - & 0.00025 \\
\hline \hline
\end{tabular}

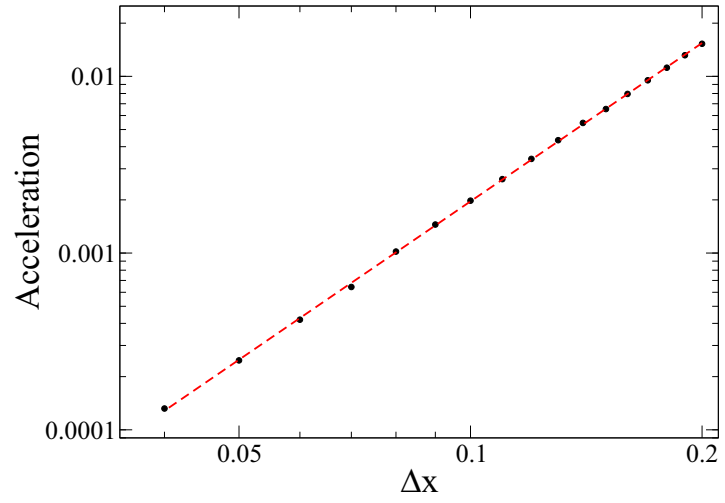

FIG. 4. Values of the proper self-acceleration $\alpha$, obtained by curve fitting the numerical results to Eq. (9). The scaling of this quantity is described well by the power law $\alpha=2 \Delta x^{3}$, shown by the dashed red line.

restriction of the wave packet. During this simulation time, the wave packet thus appeared to accelerate uniformly, which corroborates our intuition that the behavior of the discrete wave packet should approach that of the continuum case as $\Delta x \rightarrow 0$.

To put this observation on a quantitative basis, we show in Fig. 4 a logarithmic plot of the measured values of the selfacceleration as a function of the lattice discretization. The linear behavior visible clearly implies a power-law dependence of $\alpha$ on $\Delta x$. To analyze this further, and to investigate how the continuum result emerges as the lattice spacing reduces to zero, we first recall that in the continuum case $x(t)=1 / 2 a t^{2}$, where for the Airy solution we consider $a=1 / 2$. Writing the time coordinate in the lattice units of $J^{-1}$, we find that

$$
x(t)=\Delta x n(t)=\frac{1}{2} a\left(2 \Delta x^{2} t\right)^{2},
$$

where $n(t)$ is the lattice site occupied by the peak of the wave packet. The self-acceleration of the wave packet measured in lattice units is thus $\alpha=4 a \Delta x^{3}$, and so we should expect to see the power-law dependence $\alpha(\Delta x)=2 \Delta x^{3}$. We plot this curve in Fig. 4, and indeed find that it describes the scaling of $\alpha$ extremely well.

\section{Bloch oscillation}

It is interesting to compare the case of the Airy wave packet with that of a particle in a tilted lattice potential, that is, a potential which rises linearly in space, $V(x) \propto x$,

$$
H_{\mathrm{BO}}=H_{\text {latt }}+V_{0} \sum_{j} j n_{j},
$$

where $V_{0}$ is the difference in potential between neighboring sites and $n_{j}$ is the standard number operator. Naively, one could interpret the lattice tilt as resulting from the application of a constant force ( since $F=-\partial V / \partial x$ ), and so one would expect the wave packet to uniformly accelerate in the direction of the tilt. The presence of the lattice, however, complicates this simple picture; although the wave packet will initially accelerate, it will also experience Bragg diffraction from the lattice potential. The result is that it will undergo an oscillatory motion termed Bloch oscillation [16,17]. 

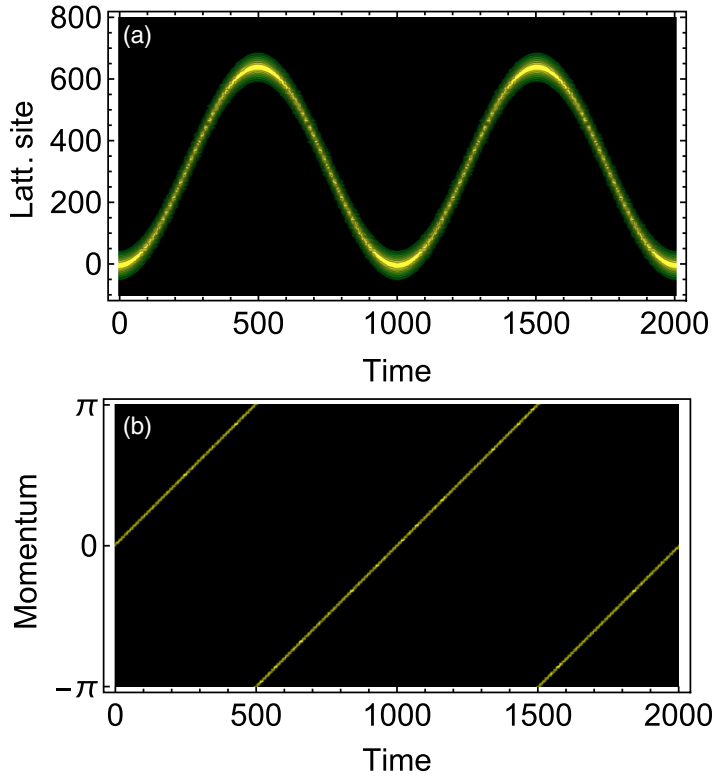

FIG. 5. Bloch oscillation of a Gaussian wave packet in a tilted lattice. (a) Probability density of the wave packet as a function of time. The wave packet makes a slow oscillatory motion (Bloch oscillation) described by Eq. (13). (b) Probability density in momentum space, $|\tilde{\psi}(k, t)|^{2}$, for the same system. The sharply peaked distribution moves linearly with time across the first Brillouin zone, crossing the boundary at $t=500$, corresponding to the reversal of motion of the wave packet in space. Parameters of the system: $\Delta x=0.2, V_{0}=2 \pi J / 1000$.

If the initial state of the particle is a well-localized wave packet, it is straightforward to show that the position of its center of mass is given by $[27,28]$

$$
x(t)=2\left(J / V_{0}\right)\left(1-\cos V_{0} t\right),
$$

where for convenience we have set the initial condition $x(t)=$ 0. In Fig. 5(a) we show the numerical simulation of a broad Gaussian wave packet under the action of the tilted lattice Hamiltonian (12). The oscillatory motion of the wave packet is clear, the amplitude and frequency of the oscillation being related to the size of the tilt. For small values of $t$, we can make a Taylor expansion of Eq. (13), to reveal that it indeed begins to accelerate uniformly, with $a=2 J / V_{0}$. As with the discretized Airy wave packet, this acceleration reduces with time, but in contrast to the Airy case it does not follow a relativistic form. Rather than asymptotically approaching the maximum lattice velocity, the wave packet instead slows and eventually turns around, and begins propagating in the opposite direction.

The difference between this behavior and that of the discretized Airy wave packet is even clearer in momentum space. In Fig. 5(b) we show the Fourier transform of the Bloch oscillation; the broad Gaussian wave packet in real space becomes a narrow Gaussian in momentum space. The effect of the lattice tilt is that the momentum distribution shifts linearly with time, $k(t)=V_{0} t$, obeying the classical equation of motion $F=\partial k / \partial t$. When the momentum reaches the edge of the first Brillouin zone at $k=\pi$, it wraps around it and reenters at $k=-\pi$, corresponding to the wave packet's motion reversing. In contrast, the momentum distribution of the Airy wave packet does not alter with time (within the Airy zone), even though we see the peaks of the wave packet appearing to accelerate along hyperbolic trajectories [29]. This underlines the importance of taking care when discussing the Airy dynamics. The Gaussian wave packet obeys Ehrenfest's theorem, and so we can consider its center of mass to be accelerated by the applied force. This is not the case for the Airy wave packet. Here the self-acceleration arises from quantum interference effects, which depend on the entire form of the wave function.

\section{E. Wave-packet manipulation}

We have seen that the critical factor determining the propagation of the lattice Airy wave packet is the maximum velocity of excitations in the lattice, $v^{\max }$. In turn this depends on the hopping parameter $J$, indicating that if we can alter $J$ coherently we will be able to finely control the trajectory of the wave packet. A powerful method to achieve this is provided by Floquet engineering [18]. In this approach, the lattice potential is periodically driven in time, or "shaken," at a frequency much higher than the other time scales of the problem. In this high-frequency limit, the full time-dependent Hamiltonian can be described by a static effective Hamiltonian with renormalized parameters. In particular, for the hopping Hamiltonian (5), the tunneling is renormalized to an effective value $J_{\text {eff }}$, and manipulating the parameters of the shaking permits the value of $J_{\text {eff }}$ to be adjusted.

We will consider the standard form of driving

$$
H(t)=H_{\text {latt }}+K \cos \omega t \sum_{j} j n_{j},
$$

in which the potential has a sinusoidal dependence on time. The behavior of this Hamiltonian was studied in Ref. [30] in the context of periodically driven semiconductor superlattices and, for the specific case of sinusoidal driving [31], the effective tunneling has the dependence

$$
J_{\text {eff }}=J \mathcal{J}_{0}(K / \omega),
$$

where $\mathcal{J}_{0}$ is the zeroth-order Bessel function of the first kind. For convenience we will use the notation $K_{0}=K / \omega$ to denote the dimensionless argument of the Bessel function. This form of driving has been used in cold atom experiments [32], and the Bessel function dependence of the tunneling has been directly observed [33,34]. The form of $J_{\text {eff }}$ is shown in Fig. 6(a). We can note that at $K_{0} \simeq 2.4048$, the first root of the Bessel function, the effective tunneling vanishes. This produces the effect known as CDT ("coherent destruction of tunneling") [35], in which the tunneling dynamics of the system is completely quenched. This effect has been used to induce the Mott transition [36,37] and to control the motion of quantum particles on lattices [38].

If we prepare the system as a lattice Airy wave packet and allow it to evolve freely, we have seen that it will undergo a relativistic acceleration with its velocity approaching arbitrarily close to the maximum lattice velocity $v^{\max }=2 \mathrm{~J}$, giving it a photonlike behavior. This can also be achieved by giving the wave packet a kick, by imprinting a phase on it of the form $\exp [i \phi j]$, where $j$ labels the lattice site, which imposes 


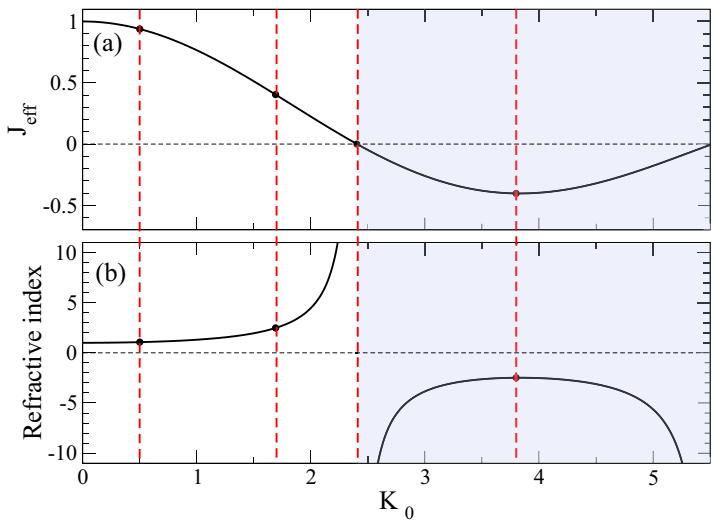

FIG. 6. (a) The effective tunneling, $J_{\text {eff }}$, for a sinusoidally driven lattice has a Bessel function dependence on the driving parameter, given by Eq. (15). The symbols mark the values of $K_{0}$ used to obtain the results shown in Fig. 7. For $K_{0}=0.5$, the amplitude of the tunneling is slightly reduced from its undriven value. At $K_{0}=1.691$, $J_{\text {eff }}=0.403$, while at $K_{0}=2.4048$ the effective tunneling vanishes. For $K_{0}>2.4048$, the shaded area, the effective tunneling is negative; at $K_{0}=3.80$ the negative effective tunneling takes its maximum value. (b) We denote the ratio of $v^{\max }$ in the undriven system to $v^{\max }$ in the driven system as the refractive index. As $K_{0}$ approaches the first zero of the Bessel function, the refractive index increases, and diverges at $K_{0}=2.4048$. In the shaded region, the refractive index is negative.

an initial velocity of $v=2 J \sin \phi$ on the wave packet. By using a value of $\phi$ close to $\pi / 2$ we can thus place the wave packet deep in the relativistic regime without waiting for it to accelerate to this state from rest. Although the results reported in this section were obtained by means of this phaseimprinting technique, we have verified that the same results are obtained by allowing the wave packet to self-accelerate to this regime.

If we consider the propagation to be photonlike, with $v^{\max }$ playing the role of the speed of light, then controlling the magnitude of $J$ gives us control of a quantity analogous to the refractive index. This will be the ratio of $v^{\max }$ in the undriven system to the maximum velocity when the lattice is shaken, which is simply given by $J / J_{\text {eff }}$. We plot this quantity in Fig. 6(b). For $K_{0}<2.4048$, the refractive index rises from its initial value of 1 , showing how the speed of light in the lattice drops. At the zero of the Bessel function, the refractive index diverges, corresponding to the system becoming infinitely optically dense. For larger values of $K_{0}$ the refractive index becomes negative, indicating that in this regime (shaded gray) negative refraction [39] occurs.

In Fig. 7 we show the probability densities for Airy wave packets under various driving conditions, obtained by the numerical simulation of the full time-dependent Hamiltonian (14). In all cases the initial amplitude of the driving was set to $K_{0}=0.5$, giving a refractive density of 1.07 , meaning that the Airy wave packet moved at a slightly lower velocity than in the absence of driving. At $t=30$ the amplitude of the driving is abruptly changed to a different value, and then restored to $K_{0}=0.5$ at $t=60$.

Figure 7(a) shows the result of reducing $J_{\text {eff }}$ to a smaller, but positive value. It can be seen that the trajectories of the peaks change their angle of propagation with respect to the $t$ axis, and the peaks continue moving along relativistic linear paths. The adjustment of their velocity to the lower value of $v^{\max }$ appears to occur essentially instantaneously, and does not cause any appreciable deformation of the wave packet's profile. When $K_{0}$ is restored to its previous value, the original form of propagation of the wave packet resumes, with the trajectories moving parallel to their original course. This behavior strongly resembles the standard refraction of light by a slab of material with a positive refractive index.

In Fig. 7(b) we show the effect of tuning $K_{0}$ to a value of 2.404, close to the zero of the Bessel function. In this case the refractive index diverges, and when the lattice is driven at this value of $K_{0}$ the motion of the wave packet is completely frozen. This is analogous to the phenomenon of slow or "stopped" light [40] previously seen in ultracold
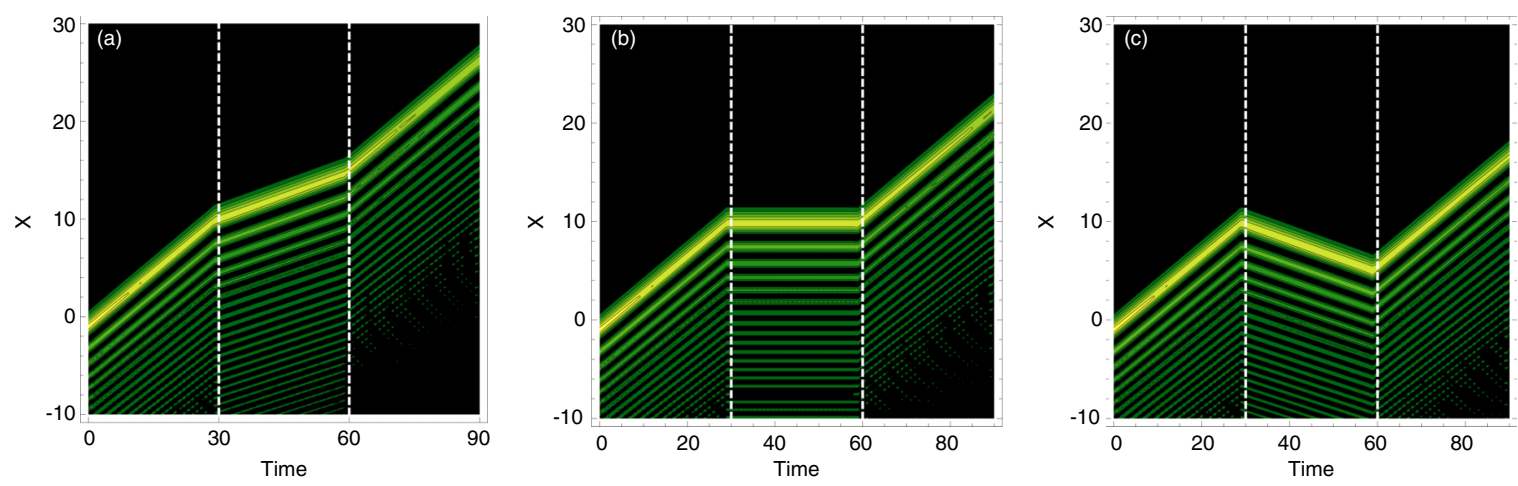

FIG. 7. Trajectories of an Airy wave packet in a driven lattice; simulation parameters: $\omega=2 \pi, \Delta x=0.2$. In all cases $K_{0}$ is initially set to a value of $K_{0}=0.5$, for which $J_{\text {eff }}$ is slightly reduced from its undriven value. At $t=30, K_{0}$ is abruptly changed to another value, while at $t=60$ it reverts to its original value; these boundaries are marked by the vertical dashed lines. (a) Between $t=30$ and $t=60, K_{0}$ is set to a value of $K_{0}=1.691$. In this time interval the velocity of the wave packet is reduced by more than half, and the peaks appear to undergo refraction at the boundaries described by Snell's law. (b) By tuning $K_{0}$ to a zero of the Bessel function $\left(K_{0}=2.4048\right)$, the wave packet's motion is frozen. This corresponds to the medium's refractive index diverging, producing an analogous effect to "stopped light." (c) Setting $K_{0}=3.80$ causes the wave packet to reverse its motion since the effective tunneling becomes negative. This behavior mimics the phenomenon of negative refraction. 


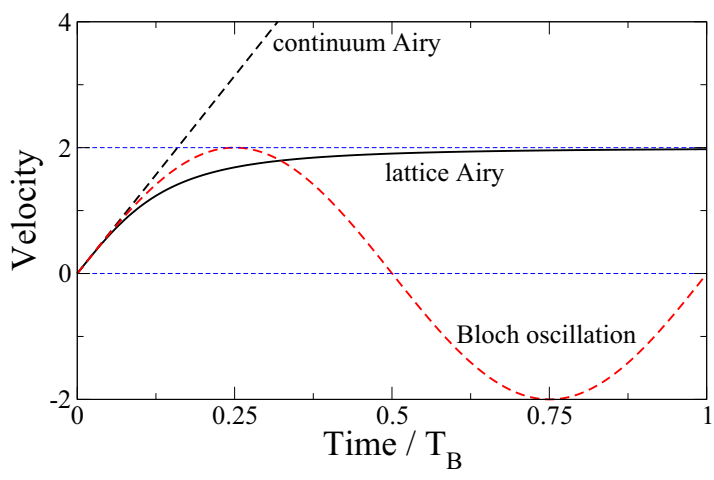

FIG. 8. Three different forms of behavior for accelerated quantum systems. The velocity of a continuum Airy wave packet rises linearly with time, and increases without limit. In contrast, in a discrete lattice system, the velocity initially rises linearly, but asymptotically approaches the value of $v^{\text {max }}$, which acts as the speed of light in this system. It should be noted that, in this case, the proper acceleration of the wave packet indeed stays constant. Finally, tilting the lattice potential subjects a wave packet to a constant uniform force. The presence of the lattice, however, means that the wave packet does not accelerate uniformly, but instead undergoes Bloch oscillation.

gases by using electromagnetically induced transparency to manipulate the refractive index. Finally, Fig. 7(c) shows the behavior of the wave packet when the effective tunneling is renormalized to a negative value. The motion of the peaks now reverses while this condition is fulfilled, mimicking the effect of a light ray traversing a region with negative refractive index.

\section{CONCLUSIONS}

We have studied the dynamics of an Airy wave packet moving in a lattice potential. Like its continuum counterpart [1], the lattice Airy wave packet undergoes self-acceleration, but due to the limitation on the maximum speed of propagation arising from the lattice structure, this acceleration reduces in time in accordance with relativistic kinematics. This contrasts with the case of a particle on a lattice subjected to a constant force. While the limited range of velocity also plays a role in this case, the particle instead follows an oscillatory motionBloch oscillation-in which its velocity periodically cycles between $\pm v^{\text {max }}$. We summarize these three different forms of motion in Fig. 8.
Lattice Airy wave packets thus provide a means to observe relativistic effects by lowering the effective speed of light to a level which is rather convenient for experiment. This opens avenues to simulate systems which are otherwise rather resistant to physical realization, such as the relativistic harmonic oscillator [41], as well as employing relativistic effects to produce more exotic effects such as enhancing the lifetimes of unstable particles via time dilation [42]. We have also shown how the trajectory of the lattice Airy wave packet can be manipulated by driving the lattice. This high level of controllability, as opposed to the single ballistic path [2] of the continuum case, makes these wave packets ideal candidates to convey matter coherently from one point in a lattice to another, with many possible applications to quantum information transfer [43]. In the deep relativistic limit, in which the wave packet's motion is photonlike, this control over the trajectory can be used to mimic a material with negative refractive index, and could in the future be used to study perfect lensing [44] of matter waves.

Finally, we turn to possible experimental implementations of this system. The driven lattice experiments of Ref. [32], for example, used a gas of approximately $10^{5}$ ultracold ${ }^{87} \mathrm{Rb}$ atoms, held in an optical lattice with a well spacing of $426 \mathrm{~nm}$ and a tunneling frequency of $J \sim 100 \mathrm{~Hz}$. This corresponds to an effective value of the speed of light of $v^{\max }=85 \mu \mathrm{m} / \mathrm{s}, 12$ orders of magnitude smaller than $c$ in free space. If we take a lattice spacing of $\Delta x=0.2$, so that the first and second peaks of the Airy wave packet are separated by 12 lattice spacings, then from Table I we can see that $\alpha=0.015$ in lattice units. This translates to $64 \mu \mathrm{m} / \mathrm{s}^{2}$ in physical units, using these values for $d$ and $J$. Thus over a time evolution of $1 \mathrm{~s}$ the wave packet would move $\sim 75$ lattice spacings, which should be easily resolvable using quantum gas microscopy $[14,45,46]$. In Ref. [8] it was noted that atomic interactions did not affect the motion of the continuum wave packet much, except in the limit of very strong interactions for which it would decay by shedding solitons. Accordingly we believe that, for weak to moderate interactions, the lattice Airy wave packet should be realizable in state-of-the-art experimental setups. Including the effects of interactions and temperature and generalizing these results to higher dimensions remain fascinating subjects for future research.

\section{ACKNOWLEDGMENTS}

This work has been supported by Spain's MINECO through Grants No. FIS2013-41716-P and No. FIS2017-84368-P.
[1] M. V. Berry and N. L. Balazs, Am. J. Phys. 47, 264 (1979).

[2] Y. Hu, P. Zhang, C. Lou, S. Huang, J. Xu, and Z. Chen, Opt. Lett. 35, 2260 (2010).

[3] J. Baumgartl, M. Mazilu, and K. Dholakia, Nat. Photon. 2, 675 (2008).

[4] P. Rose, F. Diebel, M. Boguslawski, and C. Denz, Appl. Phys. Lett. 102, 101101 (2013).
[5] S. Jia, J. C. Vaughan, and X. Zhuang, Nat. Photon. 8, 302 (2014).

[6] G. A. Siviloglou, J. Broky, A. Dogariu, and D. N. Christodoulides, Phys. Rev. Lett. 99, 213901 (2007).

[7] N. Voloch-Bloch, Y. Lereah, Y. Lilach, A. Gover, and A. Arie, Nature (London) 494, 331 (2013).

[8] N. K. Efremidis, V. Paltoglou, and W. von Klitzing, Phys. Rev. A 87, 043637 (2013). 
[9] C. Yuce, Mod. Phys. Lett. B 29, 1550171 (2015).

[10] M. Lewenstein, A. Sanpera, V. Ahufinger, B. Damski, A. Sen, and U. Sen, Adv. Phys. 56, 243 (2007).

[11] D. Jaksch and P. Zoller, Ann. Phys. (N.Y.) 315, 52 (2005).

[12] M. Aidelsburger, M. Atala, M. Lohse, J. T. Barreiro, B. Paredes, and I. Bloch, Phys. Rev. Lett. 111, 185301 (2013).

[13] H. Miyake, G. A. Siviloglou, C. J. Kennedy, W. C. Burton, and W. Ketterle, Phys. Rev. Lett. 111, 185302 (2013).

[14] Z. A. Geiger, K. M. Fujiwara, K. Singh, R. Senaratne, S. V. Rajagopal, M. Lipatov, T. Shimasaki, R. Driben, V. V. Konotop, T. Meier, and D. M. Weld, Phys. Rev. Lett. 120, 213201 (2018).

[15] M. Leder, C. Grossert, and M. Weitz, Nat. Commun. 5, 3327 (2014).

[16] C. Zener, Proc. R. Soc. London A 145, 523 (1934).

[17] F. Bloch, Z. Phys. 52, 555 (1929).

[18] A. Eckardt, Rev. Mod. Phys. 89, 011004 (2017).

[19] This corresponds to the solution given in Ref. [1] with the explicit parameter choice of $B / \hbar^{2 / 3}=1$.

[20] M. Abramowitz and I. Stegun, Handbook of Mathematical Functions (Dover Publications Inc., New York, 1970).

[21] P. Panagiotopoulos, D. Abdollahpour, A. Lotti, A. Couairon, D. Faccio, D. G. Papazoglou, and S. Tzortzakis, Phys. Rev. A 86, 013842 (2012).

[22] O. Morsch and M. Oberthaler, Rev. Mod. Phys. 78, 179 (2006).

[23] E. H. Lieb and D. W. Robinson, Commun. Math. Phys. 28, 251 (1972).

[24] E. A. Desloge and R. J. Philpott, Am. J. Phys. 55, 252 (1987).

[25] A. French, Special Relativity, M.I.T. Introductory Physics Series (Norton, New York, 1968).

[26] W. Rindler, Essential Relativity: Special, General, and Cosmological (Springer, New York, 2013).

[27] M. Holthaus, J. Opt. B 2, 589 (2000).

[28] T. Hartmann, F. Keck, H. J. Korsch, and S. Mossmann, New J. Phys. 6, 2 (2004).
[29] An alternative way of visualizing this is to see that $H_{\text {latt }}$ commutes with $k$, and so the momentum of the Airy wave packet cannot change. Introducing the tilt breaks this conservation law, and so the mean momentum is time dependent in the case of Bloch oscillation.

[30] M. Holthaus, Phys. Rev. Lett. 69, 351 (1992).

[31] C. E. Creffield, Phys. Rev. B 67, 165301 (2003).

[32] H. Lignier, C. Sias, D. Ciampini, Y. Singh, A. Zenesini, O. Morsch, and E. Arimondo, Phys. Rev. Lett. 99, 220403 (2007).

[33] C. E. Creffield, F. Sols, D. Ciampini, O. Morsch, and E. Arimondo, Phys. Rev. A 82, 035601 (2010).

[34] C. Cabrera-Gutiérrez, A. Fortun, E. Michon, V. Brunaud, M. Arnal, J. Billy, and D. Guéry-Odelin, Phys. Rev. A, arXiv:1804.02147 (unpublished).

[35] F. Grossmann, T. Dittrich, P. Jung, and P. Hänggi, Phys. Rev. Lett. 67, 516 (1991).

[36] A. Eckardt, C. Weiss, and M. Holthaus, Phys. Rev. Lett. 95, 260404 (2005).

[37] A. Zenesini, H. Lignier, D. Ciampini, O. Morsch, and E. Arimondo, Phys. Rev. Lett. 102, 100403 (2009).

[38] C. E. Creffield, Phys. Rev. Lett. 99, 110501 (2007).

[39] V. G. Veselago, Phys. Usp. 10, 509 (1968).

[40] L. V. Hau, S. E. Harris, Z. Dutton, and C. H. Behroozi, Nature (London) 397, 594 (1999).

[41] K. M. Fujiwara, Z. A. Geiger, K. Singh, R. Senaratne, S. V. Rajagopal, M. Lipatov, T. Shimasaki, and D. M. Weld, New J. Phys. 20, 063027 (2018).

[42] I. Kaminer, J. Nemirovsky, M. Rechtsman, R. Bekenstein, and M. Segev, Nat. Phys. 11, 261 (2015).

[43] L. Banchi, A. Bayat, P. Verrucchi, and S. Bose, Phys. Rev. Lett. 106, 140501 (2011).

[44] J. B. Pendry, Phys. Rev. Lett. 85, 3966 (2000).

[45] W. S. Bakr, J. I. Gillen, A. Peng, S. Fölling, and M. Greiner, Nature (London) 462, 74 (2009).

[46] C. Gross and I. Bloch, Science 357, 995 (2017). 